\title{
New Onset of Very Large Tophi in a Patient After Kidney Transplantation
}

\author{
Mohammad Hasan Alemzadeh-Ansari, MD and Mohammad Javad Alemzadeh-Ansari, MD
}

\begin{abstract}
A
woman, aged 54 years, presented to our hospital with a one-month history of painful swelling of the joints of her hands and feet, that had begun gradually over a 3-year period with no prior medical history of gouty disease. She had undergone kidney transplantation 12 years earlier and was treated with immunosuppressive medications (cyclosporine, prednisolone, and mycophenolate mofetil). Upon physical examination, she had limitation in flexion movements of the distal and proximal interphalangeal joints of both hands, and she could not bear weight due to large and painful tophi on both feet. The swollen tender joints included the distal and proximal interphalangeal joints of both hands (figure 1A), the first metatarsophalangeal joint in the right foot (figure 1B), and the first and second metatarsophalangeal joints, the transverse tarsal joint and second proximal interphalangeal joint in the left foot (figure 1C). Serum creatinine and uric acid levels were elevated (creatinine $=1.9$ $\mathrm{mg} / \mathrm{dL}$; uric acid=10.4 mg/dL), but creatinine clearance was decreased $(40.2 \mathrm{~mL} / \mathrm{min})$. A plain-film radiograph revealed extensive juxta-articular erosions with soft tissue swelling and little osteoporosis. Compensated polarized light microscopy of the lesions revealed needle-shaped, negatively birefringent urate crystals, compatible with gouty tophi. The patient was treated with celecoxib (100 mg every 12 hours), and her dose of prednisolone was increased (1 mg/kg per day). After three days she experience relief of the joint pain. On day 5, celecoxib was discontinued, and prednisolone was tapered over a 14-day period to a maintenance dose $(0.1 \mathrm{mg} / \mathrm{kg}$ per day). She was started on an escalating dose of allopurinol, beginning with $100 \mathrm{mg}$ per day until reaching $300 \mathrm{mg}$ per day. By the 6-month follow-up, she had not experienced another acute attack, and the size of the tophi had significantly decreased.
\end{abstract}

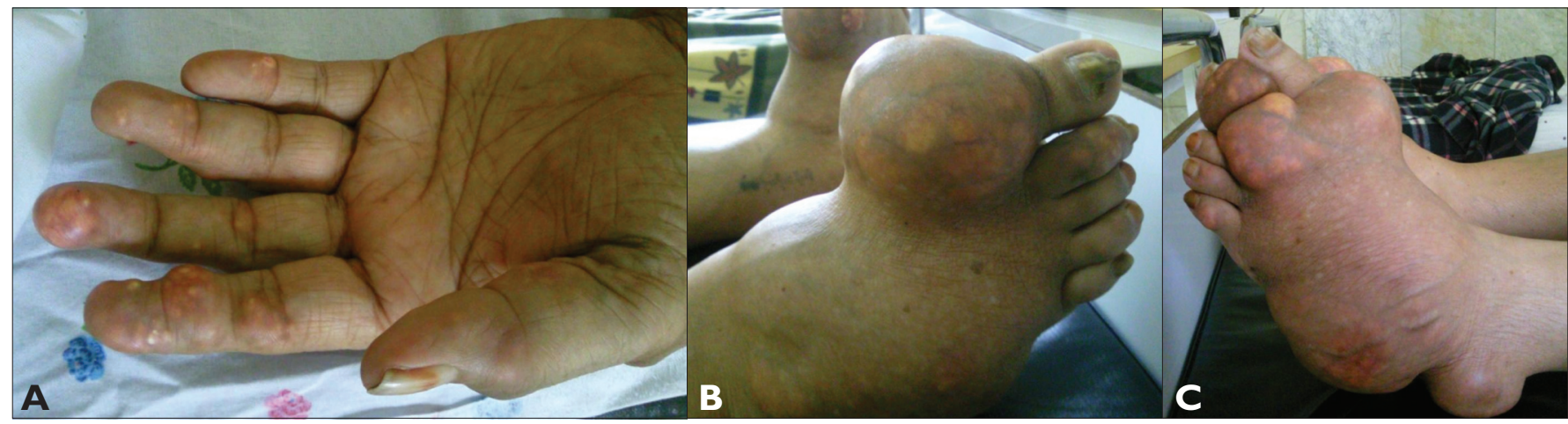

Figure 1. Multiple subcutaneous tophi are seen in the hands $(A)$ and large tophi in the feet $(B, C)$.

Corresponding Author:

Mohammad Javad Alemzadeh-Ansari, MD

Faculty of Medicine

Tehran University of Medical Sciences

Tehran, Iran

Tel: +989166174016

Email:mj.aansari@gmail.com
Keywords: Kidney transplantation;

Gout; Tophi

Received: December 10, 2010

Revised: February 2, 2011

Accepted: February 16, 2011

doi: $10.3121 / \mathrm{cmr} .2011 .982$

The Aperture, like the opening in the lens of a microscope that allows light to pass through, is a forum for art, humor, and images that provides a portal for new or different views of medicine and research. 


\section{Discussion}

Tophi are deposits of monosodium urate crystals in soft tissue that may occur in the helix of the ear, over olecranon processes, and over interphalangeal joints. Tophaceous gout may lead to significant morbidity and, if untreated, can cause joint erosion and destruction. ${ }^{1}$ Although gouty tophi are seen in chronic disease, in a few conditions tophi may be the first sign of the disorder. ${ }^{2,3}$ Hyperuricemia is a common complication in organ transplant recipients and frequently is associated with chronic cyclosporine immunosuppressive therapy. ${ }^{4,5}$ Kidney transplant recipients are prone to develop post-transplant hyperuricemia. Risk factors for hyperuricemia include decreased glomerular filtration rate, diuretic use, and pre-existent history of hyperuricemia. ${ }^{6}$ The clinical course of gout associated with organ transplantation is markedly accelerated when compared with the classic progression of primary gout. The stage of asymptomatic hyperuricemia can be present for six months to four years in cyclosporineinduced disease. The cumulative incidence of new onset gout is $7.6 \%$ at three years after kidney transplantation. ${ }^{7}$ Although gout or hyperuricemic state has been seen after kidney transplantation, and in some cases tophi were reported during treatment for gout after kidney transplantation, ${ }^{4}$ tophi as a first presentation is rare. Johnson et $\mathrm{al}^{8}$ reported a Chinese woman, aged 72 years, who was diagnosed with tophaceous gout after kidney transplantation; however, in our searches, we did not find any cases of large tophi as the first manifestation of gout after kidney transplantation.

\section{References}

1. Stamp L, Searle M, O’Donnell J, Chapman P. Gout in solid organ transplantation: a challenging clinical problem. Drugs 2005;65:2593-2611.

2. Thissen CA, Frank J, Lucker GP. Tophi as first clinical sign of gout. Int J Dermatol 2008;47:49-51.

3. Koley S, Salodkar A, Choudhary S, Bhake A, Singhania K, Choudhury M. Tophi as first manifestation of gout. Indian J Dermatol Venereol Leprol 2010;76:393-396.

4. Baethge BA, Work J, Landreneau MD, McDonald JC. Tophaceous gout in patients with renal transplants treated with cyclosporine A. J Rheumatol 1993;20:718-720.

5. Chuang SH, You ZH, Yang WC, Huang TP, Lin CC. Tophaceous gout in a renal allograft recipient. NDT Plus 2010;3:313-314

6. Mazzali M. Uric acid and transplantation. Semin Nephrol 2005;25:50-55.

7. Abbott KC, Kimmel PL, Dharnidharka V, Oglesby RJ, Agodoa LY, Caillard S. New-onset gout after kidney transplantation: incidence, risk factors and implications. Transplantation 2005;80:1383-1391.

8. Johnson S, Toms A, Lee P. Tophaceous gout: uncommon presentation of a common disease. J Rheumatol 2003; 30:863-864.

\section{Author Affiliations:}

Mohammad Hasan Alemzadeh-Ansari, MD*;

Mohammad Javad Alemzadeh-Ansari, MD'

*Faculty of Medicine, Jundishapour University of Medical Sciences, Ahvaz, Iran

†Faculty of Medicine, Tehran University of Medical Sciences, Tehran, Iran 